\title{
ADAM15 upregulation and interaction with multiple binding partners in inflammatory bowel disease
}

\author{
Jean-François Mosnier ${ }^{1}$, Anne Jarry ${ }^{1}$, Chantal Bou-Hanna ${ }^{1}$, Marc G Denis ${ }^{1}$, Didier Merlin ${ }^{2}$ \\ and Christian L Laboisse ${ }^{1}$ \\ ${ }^{1}$ INSERM, U539, Nantes, F-44035 France and ${ }^{2}$ Department of Medicine, Division of Digestive Diseases, \\ Emory University School of Medicine, Atlanta, GA, USA
}

\begin{abstract}
A disintegrin and metalloproteinase (ADAM)15 is upregulated in some tissues undergoing remodeling. This glycoprotein is characterized by adhesive function through its interaction with members of the integrin family and protease properties. The goal of this work was to describe the tissue distribution of ADAM15 and its spatial relationship with its known binding partners in inflammatory bowel disease. ADAM15 expression was examined using frozen tissues from eight patients with ulcerative colitis or Crohn's disease and four normal colons by immunohistochemistry, immunoblotting and quantitative reverse transcription-polymerase chain reaction. In addition expression of $\alpha 5 \beta 1$ - and $\alpha \mathrm{V} \beta 3$-integrins, VE-cadherin, $\alpha$-smooth mucle actin ( $\alpha$-SMA) and collagen IV was examined using immunohistochemistry and confocal microscopy. In the normal colon, ADAM15 was expressed by all epithelial cells throughout the crypt and by pericryptic myofibroblasts coexpressing $\alpha$-SMA and collagen IV. ADAM15 was also expressed by endothelial cells and vascular myocytes in all layers of the intestinal wall as well as by nonvascular myocytes of the muscularis mucosae and muscularis propria. In inflammatory bowel diseases, ADAM15 was strongly upregulated at the mRNA level and expressed only as an active form as shown by immunoblotting analysis. Parallel to its upregulation, ADAM15 expression was found both at the plasma membrane and in the cytoplasm of epithelial cells in acute attacks of the disease. In the crypt abcesses, ADAM15-positive epithelial cells were in close contact with $\alpha 5 \beta 1$-integrin-positive leukocytes localized between these cells and in the crypt lumen. In the regenerative areas, ADAM15-positive epithelial cells were in close contact with $\alpha 5 \beta 1$ - and $\alpha \mathrm{v} / 33$-positive pericryptic myofibroblasts. In endothelial cells, VE-cadherin was decreased. In contrast, ADAM15 was strongly expressed by endothelial cells and was in close contact with $\alpha 5 \beta 1$-positive leukocytes. There is a differential expression of ADAM15 in epithelial cells during inflammatory bowel disease compared with the normal colon. In addition, the spatial relationships with its binding partners suggest a role for ADAM15 in the differentiation of regenerative colonic mucosa as well as in leukocyte transmigration across epithelial and endothelial barriers.
\end{abstract}

Laboratory Investigation (2006) 86, 1064-1073. doi:10.1038/labinvest.3700465; published online 7 August 2006

Keywords: ADAM15; integrins; inflammatory bowel disease; epithelial regeneration; pericrytic myofibroblasts; tissue remodeling

ADAM15, also known as metargidin, is a member of 'a disintegrin and metalloproteinase' (ADAM) family of transmembrane glycoproteins exhibiting structural similarities with the snake venom disintegrins. ${ }^{1,2}$ The prototypical ADAM protein comprises pro, metalloprotease, disintegrin, cysteinerich, epidermal growth factor, transmembrane and cytoplasmic domains. ${ }^{1-3}$ Approximately, half of the

Correspondence: Professor J-F Mosnier, MD, PhD, INSERM U539, Faculté de Médecine, 1 Rue Gaston Veil, 44035 Nantes Cedex 1, France.

E-mail: u539@nantes.inserm.fr

Received 19 June 2006; revised 11 July 2006; accepted 12 July 2006; published online 7 August 2006
ADAMs including ADAM15 are able to function as active proteases. The disintegrin domain is involved in binding to integrins, a family of heterodimeric membrane glycoproteins that play a role in cell adhesion, migration and signal transduction. Like the disintegrin domain, the region rich in cysteine residues is also implicated in adhesion. The disintegrin and cystein-rich sequences may represent a functional entity, that is, the adhesion domain. ${ }^{4}$ The cytoplasmic tail can vary widely in size among the different ADAMs. In this domain, ADAM15 is rich in proline and serine residues and thus may interact with Src homology 3 domains in intracellular proteins. ${ }^{5}$ Some ADAMs like ADAM15 also undergo tyrosine phosphorylation and interact with 
cytoplasmic proteins containing Src homology 2 binding sites. The interaction with these intracellular proteins suggest that ADAM15 may be involved in intracellular signalling. ${ }^{5,6}$

ADAM15 was shown to be upregulated in tissues undergoing remodeling such as in developing lesions of osteoarthritis and atherosclerosis. ${ }^{7-9}$ In several 'in vitro' models, migration can be partially blocked by ADAM15 antibodies, antisense ADAM15 oligonucleotides and the metalloprotease inhibitor BB-3103. ${ }^{10}$ In vitro experimental studies have highlighted ADAM15 involvement in cell-cell interactions, thus pointing its proeminent adhesive function. However, human ADAM15 appears as an adhesion molecule with unique properties for several reasons. It contains a RGD (arginin-glycinaspartic acid) sequence in the predicted integrinbinding loop of its disintegrin domain. ${ }^{11}$ ADAM15 binds to $\alpha 5 \beta 1$ - and $\alpha \mathrm{v} \beta 3$-integrins through its RGD sequence $^{12}$ but also to $\alpha 9$-chain in a RGD-independent manner via a conserved sequence RX(6)DLPEF (arginin-X(6)-aspartic acid-leucine-proline-glutamic acid-phenylalanine). ${ }^{13}$ ADAM15 is partially colocalized with VE-cadherin in adherens junction. $^{14}$ Finally, ADAM15 can degrade type IV collagen and gelatin 'in vitro', through its metalloprotease activity. ${ }^{10}$ Thus, ADAM15 is a good candidate to play a pivotal role in pathological processes involving intense tissue remodeling via cell-cell and cell-extracellular matrix interactions, such as inflammatory bowel disease. However, the critical question to how the interactions revealed 'in vitro' relate to the pathological changes of inflammatory bowel disease 'in vivo' has remained unanswered.

Inflammatory bowel disease includes two chronic intestinal disorders, ulcerative colitis and Crohn's disease. Once established, patients with inflammatory bowel disease suffer episodic acute attacks that become superimposed on chronic disease. An early feature of active colitis is the formation of cryptitis, which evolves to crypt abcesses and crypt ulcers that resolve spontaneously or in response to therapy. During this healing phase, the epithelium actively regenerates. Epithelial regeneration extends from the base of the crypts and from the edge of the ulcers. Owing to the repeated episodes of the ulceration followed by the periods of healing, mucosal dystrophy becomes more and more complex. ${ }^{15}$ In this context, it is well known that the expression of several adhesion molecules is sequentially modified during the successive phases of mucosal injury and regeneration. ${ }^{16-18}$

The present report, based on colonic tissue samples from patients with inflammatory bowel disease and control subjects, describes the tissue distribution of ADAM15 and integrates its spatial relationship with its binding partners and other major adhesion molecules in the pathophysiology of inflammatory bowel disease.

\section{Materials and methods}

\section{Patients}

Four patients with ulcerative colitis and four patients with Crohn's disease having undergone a colectomy were studied. The patients with ulcerative colitis consisted of two women and two men (mean age $=35$ years; range $=22-45$ years). The patients with Crohn's disease consisted in three women and one man (mean age $=46$ years; range $=32-72$ years). Ulcerative colitis and Crohn's disease were diagnosed on clinical, endoscopic and histological criteria. The main characteristics of diseases are summarized in Table 1. In parallel studies, normal mucosae of four patients having undergone colectomy for colorectal carcinoma were studied as controls. Several samples of colon were snap-frozen in liquid nitrogen. These samples were used for determining expression of adhesive molecules in particular ADAM15 and its potential binding partners by immunohistochemistry, ADAM15 activation by immunoblot analysis, and ADAM15 mRNA expression by quantitative reverse transcription-polymerase chain reaction (RT-PCR). This study was performed in accordance with local ethical guidelines. Snap frozen tissues were collected by the Department of Pathology, belonging to the BioBank Network of Institut Régional du Cancer Nantes-Atlantique, according to the guidelines of the French Ethics Committee for Research on human tissue. ${ }^{19}$

\section{Pathological Analysis}

Hematoxylin and eosin stained frozen sections were reviewed for the presence of chronic colitis and degree of disease activity. The histological findings are summarized in Table 1.

\section{Immunoperoxidase and Immunofluorescence on Frozen Tissue Sections}

Serial sections from frozen tissue blocks were cut and stained with the primary monoclonal antibodies using a three-step immunoperoxidase technique, as described by Mason et al. ${ }^{20}$ Four micrometer-thick sections were dried overnight at room temperature and fixed for $10 \mathrm{~min}$ in acetone immediately before use. After rehydratation in TBS, tissue sections were sequentially incubated for $40 \mathrm{~min}$ with peroxidaselabeled species-specific immunoglobulin antibodies. Peroxidase-labeled species-specific immunoglobulins (DakoCytomation, Glostrup, Denmark) were diluted 1:20 in TBS. The color reaction product was developed according to the method of Graham and Karnovsky. ${ }^{21}$ A light nuclear countercoloration was obtained by staining sections with Harris' hematoxylin for $60 \mathrm{~s}$. The following antibodies were used: ADAM15 (polyclonal, dilution 1:50, R\&D 
Table 1 Clinical data, localization of lesions, morphological changes observed in frozen samples, and relative expression of ADAM15 in eight inflammatory bowel disease and three normal colonic controls

\begin{tabular}{|c|c|c|c|c|c|}
\hline$\#$ & & $\begin{array}{l}\text { Indication for surgical } \\
\text { treatment }\end{array}$ & Location & $\begin{array}{l}\text { Morphological changes observed in frozen } \\
\text { samples }\end{array}$ & $\begin{array}{l}\text { Ratio ADAM15/ } \\
\beta \text {-actin } \\
\text { transcripts }\end{array}$ \\
\hline 1 & $\mathrm{CD}$ & $\begin{array}{l}\text { Failure of medical } \\
\text { treatment }\end{array}$ & $\begin{array}{l}\text { Small bowel } \\
\text { Right colon }\end{array}$ & $\begin{array}{l}\text { Regenerative changes } \\
\text { Moderate inflammatory infiltrate } \\
\text { Lymphoid aggregates }\end{array}$ & $\begin{array}{l}\mathrm{S} 1=726266 \\
\mathrm{~S} 2=1524\end{array}$ \\
\hline 2 & $\mathrm{CD}$ & Stenosis and failure & $\begin{array}{l}\text { Left colon } \\
\text { Rectum }\end{array}$ & $\begin{array}{l}\text { Regenerative changes } \\
\text { Severe inflammatory infiltrate } \\
\text { Lymphoid aggregates }\end{array}$ & Not done \\
\hline 3 & $\mathrm{CD}$ & Fistule & $\begin{array}{l}\text { Ileum } \\
\text { Right colon }\end{array}$ & $\begin{array}{l}\text { Regenerative changes } \\
\text { Mild inflammatory infiltrate }\end{array}$ & Not done \\
\hline 4 & $\mathrm{CD}$ & $\begin{array}{l}\text { Failure of medical } \\
\text { treatment, } \\
\text { Fistule }\end{array}$ & $\begin{array}{l}\text { Ileum } \\
\text { Left colon }\end{array}$ & $\begin{array}{l}\text { Regenerative changes } \\
\text { Severe inflammatory infiltrate } \\
\text { Lymphoid aggregates } \\
\text { Ulcerative changes }\end{array}$ & $\begin{array}{l}\mathrm{S} 1=8949 \\
\mathrm{~S} 2=176493\end{array}$ \\
\hline 5 & UC & $\begin{array}{l}\text { Failure of medical } \\
\text { treatment }\end{array}$ & Pancolitis & $\begin{array}{l}\text { Regenerative changes } \\
\text { Severe inflammatory infiltrate }\end{array}$ & $\begin{array}{l}\mathrm{S} 1=4318 \\
\mathrm{~S} 2=16800\end{array}$ \\
\hline 6 & UC & $\begin{array}{l}\text { Failure of medical } \\
\text { treatment }\end{array}$ & $\begin{array}{l}\text { Left colon } \\
\text { Rectum }\end{array}$ & $\begin{array}{l}\text { Regenerative changes } \\
\text { Moderate inflammatory infiltrate }\end{array}$ & $S=54268$ \\
\hline 7 & UC & $\begin{array}{l}\text { Failure of medical } \\
\text { treatment }\end{array}$ & $\begin{array}{l}\text { Left colon } \\
\text { Rectum }\end{array}$ & $\begin{array}{l}\text { Regenerative changes } \\
\text { Moderate inflammatory infiltrate } \\
\text { Lymphoid aggregates } \\
\text { Ulcerative changes }\end{array}$ & $S=6166$ \\
\hline 8 & UC & $\begin{array}{l}\text { Failure of medical } \\
\text { treatment }\end{array}$ & Pancolitis & $\begin{array}{l}\text { Regenerative changes } \\
\text { Moderate inflammatory infiltrate } \\
\text { Lymphoid aggregates }\end{array}$ & Not done \\
\hline & NT & Colon cancer & & Normal & $\begin{array}{l}\mathrm{S} 1=5 \\
\mathrm{~S} 2=3 \\
\mathrm{~S} 3=1\end{array}$ \\
\hline
\end{tabular}

CD: Crohn's disease; UC: ulcerative colitis; NT: normal tissue; S: sample.

systems, Mineapolis, MN, USA), VE-cadherin (clone BV6, dilution 1:10, Chemicon, Temecula, CA, USA), $\alpha 5 \beta 1$-integrin (clone P1D6, dilution 1:25, DakoCytomation), $\alpha \mathrm{v} \beta 3$-integrin (clone LM142, dilution 1:50, Chemicon), $\alpha$-smooth muscle actin ( $\alpha$-SMA), (clone 1A4, dilution 1:100, Dako Cytomation) and collagen IV (clone CIV22, dilution 1:200, Dako Cytomation).

For coexpression studies, double immunofluorescence followed by confocal microsocopy were performed on $7 \mu \mathrm{m}$, paraformaldehyde-fixed or acetone-fixed frozen sections of four normal colon samples, as previously reported. ${ }^{22}$ The following double staining combinations were performed: ADAM15 (goat, 1:50)/ $\alpha$-SMA (rabbit, 1:200, Abcam, Oxon, UK); ADAM15/collagen IV (mouse/1:100); $\alpha 5 \beta 1$ (mouse, 1:25)/ $\alpha$-SMA; $\alpha \mathrm{v} \beta 3$ (mouse, 1:50)/ $\alpha$-SMA. Briefly, sections were incubated for $1 \mathrm{~h}$ in a mixture of primary antibodies, and then for $30 \mathrm{~min}$ with a mixture of alexa fluor 488- or 568-conjugated anti-mouse, anti-rabbit or anti-goat antibodies as required for a given antibodies combination (1:200, Molecular Probes, Eugene, OR, USA). Nuclear staining was performed with TOPRO-3 $(1 \mu \mathrm{M}$, Molecular Probes). Negative controls by omission of the primary antibodies were performed in each experiment. Sections were then mounted using Prolong antifade medium (Molecular Probes), and examined with a $\times 63 / 1.4$ oil objective lens on a Leica TCS-SP confocal laser-scanning microscope
(Leica, Heidelberg, Germany), using the TCS/NT software (Leica).

\section{Immunoblot Analysis}

For total extraction, samples taken from the eight patients with ulcerative colitis or Crohn's disease and one sample of normal colon were lysed in RIPA buffer supplemented with protease inhibitors and centrifuged as previously described. ${ }^{22}$ Proteins $(50 \mu \mathrm{g})$ were separated by electrophoresis on $10 \%$ polyacrylamide gels and transferred onto nitrocellulose membranes $(0.45 \mu \mathrm{m}$ porosity, Bio-Rad, Hercules, CA, USA) using Trans-blot apparatus (Bio-Rad). After blocking, membranes were incubated with a goat polyclonal antibody against human ADAM15 cytoplasmic tail (dilution 1:100; R\&D systems) or with a mouse monoclonal antibody against human $\beta$-actin (dilution 1:10000; Sigma), and then with a horseradish-peroxidase conjugated, rabbit anti-goat antibody (dilution 1:20 000; Biodesign Int., Kennebunk, ME, USA) or goat antimouse antibody (dilution 1:1000; Santa Cruz Biotechnology, Santa Cruz, CA, USA). The immunoreactive proteins were detected on films using an enhanced chemiluminescence substrate according to the manufacturer's instructions (Roche Diagnostics, Meylan, France). 


\section{RNA Extraction and Quantitative RT-PCR}

Total RNA was extracted from eight samples of five of the eight patients with inflammatory bowel disease using TRIZOL reagent (Invitrogen, Cergy Pontoise, France) and the Fast Prep cell disrupter (Qbiogen, Illkirch, France), after histological analysis of inflammatory lesions. Three colonic samples taken at distance of tumor in patients with colon carcinoma served as normal controls. RNA $(5 \mu \mathrm{g})$ was denatured at $72{ }^{\circ} \mathrm{C}$ for $3 \mathrm{~min}$ and then reversetranscribed for $60 \mathrm{~min}$ at $42^{\circ} \mathrm{C}$ in a $25 \mathrm{ml}$ volume reaction volume $(50 \mathrm{mM}$ Tris $\mathrm{HCl}, \mathrm{pH} 8.3 ; 75 \mathrm{mM}$ $\mathrm{KCl} ; 3 \mathrm{mM} \mathrm{MgCl}_{2} ; 10 \mathrm{mM}$ DTT) containing $0.5 \mu \mathrm{g}$ of random hexamers (Promega, Charbonnières, France), dNTPs (1 mM each), Rnasin (50 units) and Rnase H-MMLV reverse transcriptase (200 units).

ADAM15 and $\beta$-actin mRNA were quantified using commercially available kits (TaqMan Gene Expression Assays Hs00187052 and Hs99999903, respectively; Applied Biosystems, Courtaboeuf, France) according to the manufacturer's recommendations. Amplifications were performed in a 7700 thermocycler (Applied Biosystems).

External standard curves were generated with serial fivefold dilutions of pooled cDNA samples. The reference curve was constructed by plotting the relative amounts of these dilutions vs the corresponding $C_{\mathrm{t}}$ (threshold cycle) values. The correlation coefficient of these curves was always greater than 0.99. The relative amount of ADAM15 and $\beta$ actin transcripts was calculated from these standard curves using the ABI Prism 7000 SDS software. Samples were tested in duplicate and the average values were used for quantification as previously described. ${ }^{22}$ For each sample, the ratio between the relative amount of ADAM15 and $\beta$-actin transcripts was then calculated to compensate for variation in the quantity and quality of starting mRNA as well as for differences in reverse transcriptase efficiency.

\section{Results}

\section{Immunolocalization of ADAM15 and Its Binding Partners}

Normal colon

In the mucosa, ADAM15 was expressed by all epithelial cells lining the crypt and the surface and was localized mainly at the baso-lateral membrane of the cells (Figures 1 and 2a, b) and also in the cytoplasm with a dot-like pattern (Figure 2a and b). ADAM15 was also expressed by pericryptic cells, likely myofibroblasts and all the vessels running in the mucosa, submucosa and muscularis propria. ADAM15-positive cells included endothelial and smooth muscle cells around the veinular and arterial endothelium as well as rare resident mononuclear inflammatory cells of the lamina propria. Double immunofluorescence followed by confocal microscopy confirmed that $\alpha$-SMA-positive and collagen IV-positive pericryptic myofibroblasts expressed ADAM15 (Figure 2a and b). Finally, all the smooth muscle cells of the muscularis mucosae and muscularis propria were ADAM15-positive (Figure 1). Myenteric and submucosae plexus did not express ADAM15.

In the mucosa, some spindle cells lining the basal membrane of the crypts, essentially at their base, expressed $\alpha 5 \beta 1-$ and $\alpha \mathrm{v} \beta 3$-integrins. These cells were identified as pericryptic myofibroblasts using double immunostaining for $\alpha$-SMA (Figure 2c and d). $\alpha 5 \beta 1$ - and $\alpha \mathrm{v} \beta 3$-integrins were also expressed by the endothelial cells lining the vessels and the smooth muscle cells of muscularis mucosae (Figure 2c and d).

\section{Inflammatory bowel disease}

On microscopic examination, all the samples available for immunohistochemistry showed lesions associated with acute onset of the inflammatory bowel disease. In the mucosa, these lesions included an inflammatory infiltrate around the vessels, crypt abcesses, ulcerations and regenerative changes. Although sequentially appearing, all these lesions were simultaneously observed in each case studied.

The inflammatory infiltrate of the digestive wall and all around the ulcerations comprised a considerable number of scattered leukocytes. Some polymorphonuclear and mononuclear cells expressed ADAM15 and $\alpha 5 \beta 1$ (Figure $3 \mathrm{a}$ and b). The vascular compartment plays a pivotal role in inflammation. Like in the normal colon, ADAM15 was strongly expressed by endothelial cells of all the vessels in all samples of inflammatory bowel disease (Figure 3b). This expression was also observed in the newly formed vessels, localized in close contact to ulcerative changes of inflammatory bowel disease. In this context of acute inflammation, the immunophenotype of endothelial cell included the ADAM15 expression (Figure 3b) together with that of $\alpha 5 \beta 1$-integrin (Figure 3a), $\alpha \mathrm{v} \beta 3$-integrin, VE-cadherin (Figure $3 \mathrm{~d}$ ). In the acute attacks of ulcerative colitis, there was a focal loss of VEcadherin expression by endothelial cells in congestive and ectatic capillaries (Figure 3d) compared with that of normal colon (Figure 3c).

In crypt abcesses, ADAM15 was expressed at the plasma membrane and within the cytoplasm of epithelial cells. Only rare epithelial cells lining crypt abcesses expressed $\alpha 5 \beta 1$. In cryptitis lesions and crypt abcesses, some intraepithelial polymorphonuclear cells expressed $\alpha 5 \beta 1$-integrin.

In the regenerative mucosa characterized by epithelial mucin depletion and a severe inflammatory infiltrate, ADAM15 was strongly expressed by the epithelium as shown by immunohistochemistry (Figure 4a). In the regenerative epithelial cells, ADAM15 was expressed at the plasma membrane and within the cytoplasm with a diffuse pattern. This immunohistochemical pattern differed from that of the unaffected mucosa, particularly in 

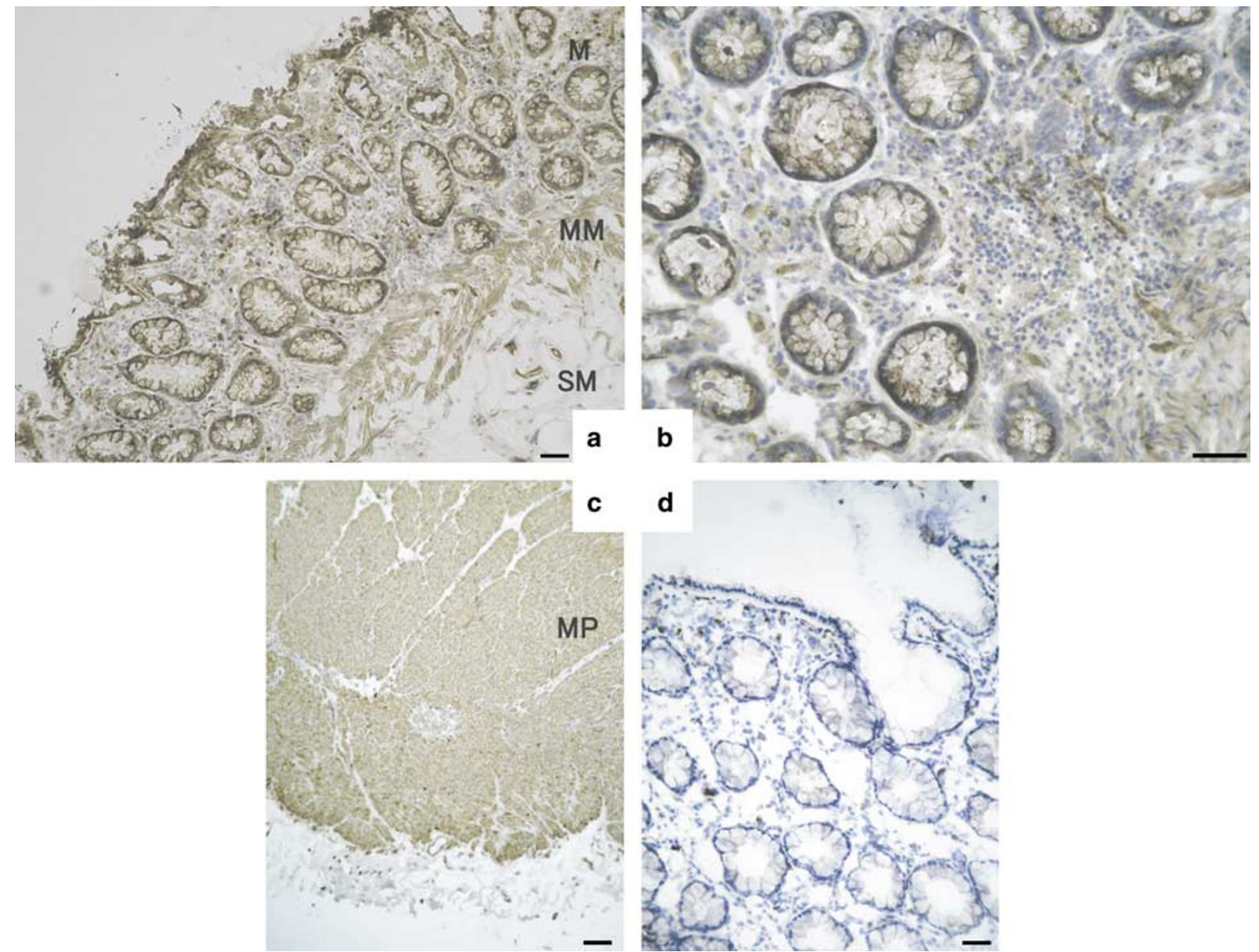

Figure 1 Normal colon. (a) A strong expression of ADAM15 by colonic epithelial cells and endothelial cells of the mucosa (M) and submucosa (SM). ADAM15 is weakly expressed by smooth muscle cells of the muscularis mucosae (MM); (b) ADAM15-positive staining at the basolateral membrane of normal epithelial cells; (c) ADAM15 immunostaining of the muscularis propria (MP); (d) negative control. Scale bars: $100 \mu \mathrm{m}$.

Crohn's disease with segmental involvement where ADAM15 expression was restricted to the basolateral plasma membrane of epithelial cells. $\alpha 5 \beta 1$ Integrin was strongly and constantly expressed by pericryptic spindle cells lining regenerative crypts, identified as $\alpha$-SMA-positive pericryptic myofibroblastic cells in double immunostaining experiments (not shown). These cells appeared more numerous than in normal colon.

Immunoblotting analysis was performed to examine whether intestinal inflammation is paralleled by changes in the pattern of ADAM15 activation. First, we found that the normal colon examined expressed only the activated form of ADAM15. This pattern remained unchanged in Crohn's disease and ulcerative colitis (Figure 4b).

Quantitative RT-PCR analysis was performed to test whether overexpression of ADAM15 occurred at the mRNA level in inflammatory bowel disease. Eight samples of only five of the patients with inflammatory bowel disease were available for this study. ADAM15 mRNAs were strongly overex- pressed in all the eight samples tested compared with three normal colonic tissues which served as controls (Table 1).

\section{Discussion}

This study is the first to morphologically assess the distribution of ADAM15 in normal and pathological settings of the digestive tract. Our findings argue for the involvement of ADAM15 in cell-cell adhesion governing the migration and regenerative mechanisms.

First, we observed a robust ADAM15 upregulation at the mRNA level, together with a change in the pattern of epithelial ADAM15 expression in inflammatory bowel disease in association with regenerative and architectural alterations. In immunoblot blot analysis, ADAM15 was only present as an activate form as previously described in mouse tissues $^{23}$ and normal human colon. ${ }^{24}$ While ADAM15 was mainly localized at the plasma 

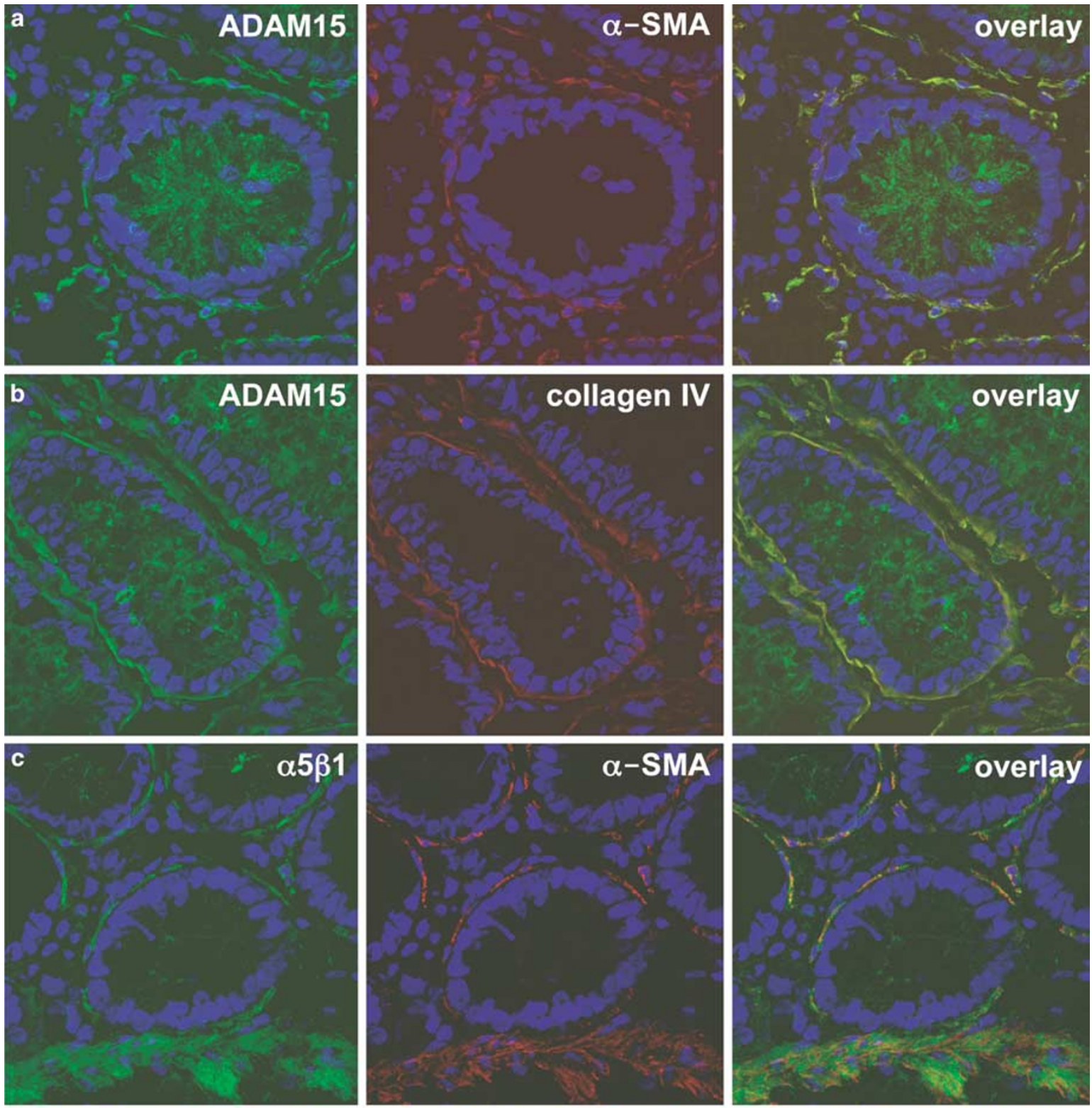

\section{d}

avß3
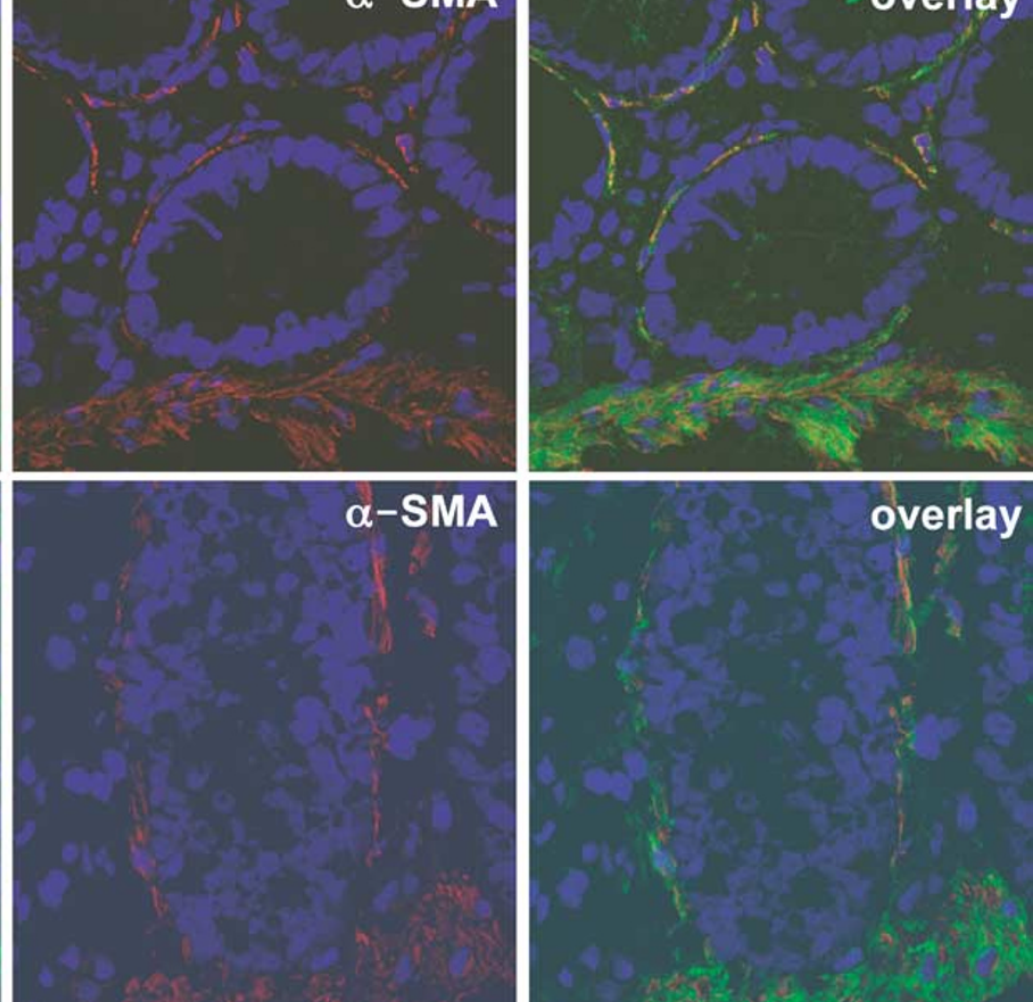

Figure 2 Normal colon. (a, b) Double immunostaining of ADAM15 (green), and $\alpha$-SMA (red, a) or collagen IV (red, b), was performed on paraformaldehyde-fixed frozen sections and examined by confocal microscopy, as described in Materials and methods. Nuclei appear in blue. ADAM15 is strongly expressed by epithelial cells, and by pericryptic myofibroblasts, which coexpress $\alpha$-SMA and collagen IV. (c, d) Double immunostaining of $\alpha 5 \beta 1$ (green, $\mathbf{c}$ ) or $\alpha \mathrm{v} \beta 3$ (green, $\mathbf{d}$ ) and $\alpha$-SMA (red) was performed on acetone-fixed frozen sections and examined by confocal microscopy, as described in Materials and methods. Nuclei appear in blue. Pericryptic myofibroblasts, scoring positive for $\alpha$-SMA, strongly express $\alpha 5 \beta 1$ and $\alpha \mathrm{v} \beta 3$, as well as muscularis mucosae myocytes. Scale bars: $20 \mu \mathrm{m}$. 

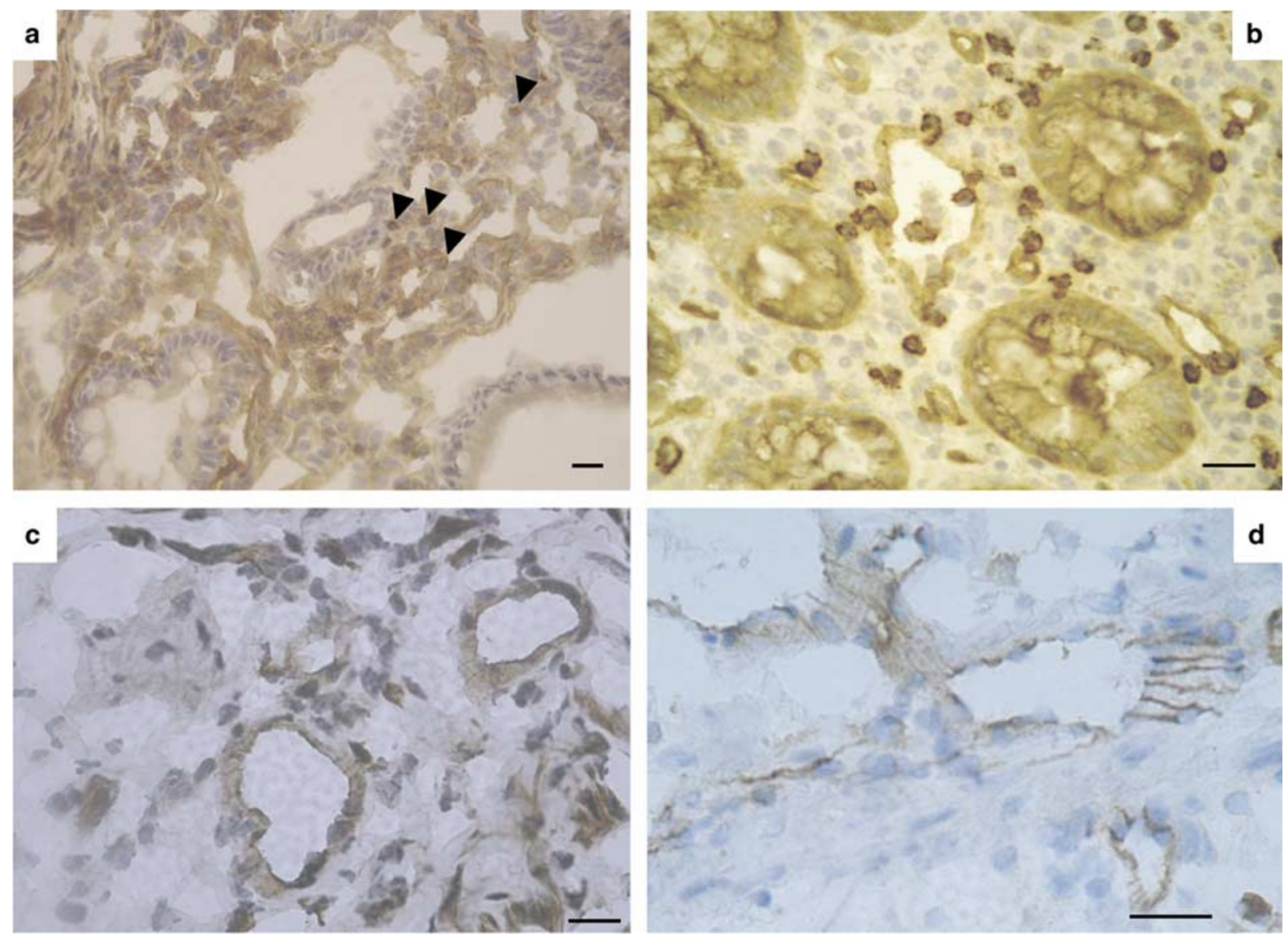

Figure 3 Inflammatory bowel disease. (a) Strong expression of $\alpha 5 \beta 1$-integrin by pericryptic myofibroblasts and polymorphonuclear cells (arrowheads) (original magnification $\times 200$ ); (b) Polymorphonuclear cell diapedesis through endothelial cells expressing ADAM15; (c and d) VE-cadherin expression is decreased at the membrane of endothelial cells (d), as compared with normal control (c). Scale bars: $50 \mu \mathrm{m}$.

membrane in the normal superficial and crypt epithelium, it was also localized within the cytoplasm of epithelial cells in inflammatory bowel disease. Our results support the concept already put forward by Lum et $a^{23}$ of ADAM15 intracellular compartmentalization, and argue that the intracellular accumulation of ADAM15 in regenerative/ undifferentiated cells corresponds to a maturation compartment while its localization at the cell membrane occurs when it engages cell surface receptors of neighbouring cells.

The second major finding is the delineation of cellular crosstalks (1) between epithelial and pericryptic myofibroblastic cells via ADAM15- $\alpha 5 \beta 1$ or ADAM15- $\alpha \mathrm{v} \beta 3$ integrins interactions, (2) between endothelial or epithelial cells and inflammatory mononuclear cells via ADAM15- $\beta 1$ interactions through RGD-binding ligands of polymorphonuclear cells and ADAM15.

An interesting finding of our study is the spatial relationship between the ADAM15-positive epithelial cells lining the crypts and $\alpha 5 \beta 1-$ and $\alpha \mathrm{v} \beta 3$ integrin-positive pericryptic myofibroblasts, both in normal tissue and regenerative mucosa in inflam- matory bowel disease. As the disintegrin domain of human ADAM15 has a conserved RGD sequence and associates with $\alpha 5 \beta 1$ - or $\alpha \mathrm{v} \beta 3$-integrins in a RGD-dependent manner, ${ }^{12}$ our observations thus suggest a potential crosstalk between regenerative epithelial cells and pericryptic myofibroblasts through an ADAM15- $\alpha 5 \beta 1$ or $\alpha \mathrm{v} \beta 3$-integrin binding. In this context, it is worth noting that subepithelial myofibroblats are of critical importance in epithelial-mesenchymal interactions and that there is a significant paracrine crosstalk during epithelial differentiation. For example, several growth factors, such as hepatocyte growth factor, keratinocyte growth factor and transforming growth factor $\beta$, whose receptors are located on epithelial cells, are secreted by myofibroblasts. ${ }^{25-27}$ It must be pointed out that the healing process resides mainly in the ability of mesenchymal cells to migrate through granulation tissue at ulcer edges and heal the ulcer bed before re-epithelialization and restoration of barrier function. ${ }^{28}$ Recently, bone marrow derivation of pericryptic myofibroblasts of human small intestine and colon has been reported. ${ }^{29}$ Colonic epithelial cells undergo cell cycle arrest, lineage-specific 


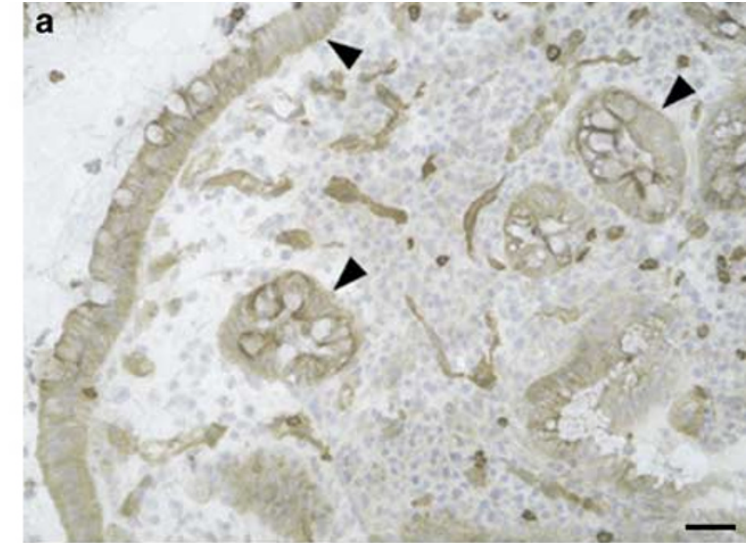

b

1

2

3

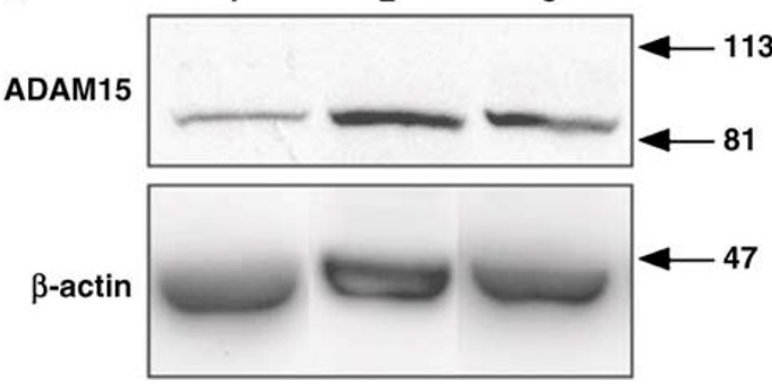

Figure 4 Inflammatory bowel disease: regenerative changes, (a) immunohistochemistry: Strong expression of ADAM15 in regenerative cryptic epithelial cells with mucus depletion (arrowheads) (Scale bar: $50 \mu \mathrm{m}$ ); (b) immunoblot: A representative immunoblot shows that the active form of ADAM15 protein $(90 \mathrm{kDa})$ is expressed in normal colon, as well as in ulcerative colitis and in Crohn's disease. Immunoblot analysis was performed as described in Materials and methods from cell lysates of human colon (lane 1), of ulcerative colitis (lane 2) and of Crohn's disease (lane 3) (50 $\mu \mathrm{g}$ protein loaded) using polyclonal goat antiADAM15 ectodomain or anti- $\beta$ actin antibodies. Numbers on the right are the molecular size of standards in thousands.

differentiation and apoptosis as they migrate along the crypt axis to reach the luminal surface. The increased expression of extracellular matrix-associated genes suggests that increased deposition of an extracellular matrix may be a function of differentiated colonic epithelial cells. In this context, our results suggest that ADAM15 through its interaction with $\alpha 5 \beta 1$-integrin could favor the differentiation of colonic epithelial cells in the regeneration course. This hypothesis is consistent with several recent experimental results: (i) Herren et $a l^{30}$ have shown that ADAM15 overexpression in the fibroblastic cell line NH3T3 inhibited cell migration; (ii) ADAM15 overexpression in $\mathrm{CaCO}_{2}-\mathrm{BBE}$ monolayers inhibited the wound repair process in wound-healing experiments using the ECIS technology. ${ }^{24}$ In this latter work, it was already suggested that ADAM15 could inhibit cell migration by interacting with integrins and therefore balancing interactions between integrins and extracellular matrix.

Our demonstration of a strong expression of ADAM15 by endothelial cells suggests a role for
ADAM15 in leukocyte-endothelial cells transmigration associated with acute inflammatory changes in inflammatory bowel disease. In fact, there are many inflammatory cells expressing $\alpha 5 \beta 1$-integrin in close contact to the vessels in the inflamed tissues. As the ADAM15 ectodomain contains an RGD sequence, it is possible that heterophilic interactions between ADAM15 and leukocytes facilitates their transmigration across the capillaries. ${ }^{31}$

Our study gives clues as to the role of ADAM15 in leukocyte transmigration across the endothelial barrier through (1) the heterophilic binding of ADAM15 with leukocyte integrins and (2) its colocalization with VE-cadherin. The process of leukocyte transmigration across the endothelial barrier has been subdivided into the sequential steps of tethering, rolling, adhesion, diapedesis and migration across the basal lamina, each controlled by different molecules. In an assay that distinguishes the role for a molecule in adhesion (ie tethering, rolling and adhesion to the apical surface of endothelial cells) vs transmigration (ie diapedesis and migration across the basal lamina), leukocyte integrins were found to have no specific role in diapedesis. They were involved only in adhesion to the apical surface of endothelial cells. ${ }^{32}$ Whereas tethering, rolling, adhesion on the apical surface all involve heterophilic interactions between one class of molecule on the leukocyte and another class of molecule on the endothelial cell, ADAM15 being a candidate for such an interaction, molecular interactions of major importance to diapedesis could rather involve homophilic interaction between a molecule on the leukocyte and the same molecule on the endothelium at the junctions. CD31 and CD99 are the best examples of this concept. In this context, this could mean that ADAM15 is involved only in the early steps of leukocyte transmigration. However, it has been recently reported that ADAM15 is colocalized with VE-cadherin in adherens junctions of endothelial cells. ${ }^{13}$ Here, we show that ADAM15 is constantly and strongly expressed by endothelial cells in inflammatory areas of inflammatory bowel diseases, whereas the VE-cadherin expression decreases. This latter finding agrees with an 'in vitro' study performed under flow condition, which has demonstrated that VE-cadherin is transiently removed from the junctions during diapedesis. Adherens junctions appear to disorganize during transmigration of monocytes ${ }^{33}$ supporting the concept that integrity of the adherens junction is a barrier to leukocyte migration. As ADAM15 is a component of adherens junctions, the question arises of why its expression does not decrease during chronic inflammation in parallel to that of VE-cadherin. A response may be that ADAM15 participates in diapedesis and migration across basal lamina not only through heterophilic interactions with leukocyte integrins, but also by collagen IV and gelatin lysis through its metalloprotease activity. Our hypothesis therefore questions the 
concept postulating that diapedesis is dependent only on homophilic interactions.

Disease activity in inflammatory bowel disease is linked to an influx of neutrophils into the mucosal epithelium (cryptitis) that can result in the formation of crypt abcesses. In vitro studies using human intestinal cell lines have shown that neutrophil migration across epithelial monolayers occurs via a paracellular route. ${ }^{34}$ Thus, it may be also hypothesized that ADAM15 participates in the migration of leukocytes across the epithelium. Indeed, there is a strong expression of ADAM15 by epithelial cells lining the crypts. As E-cadherin is decreased in the lateral membranes of epithelial cells directly exposed to transmigrating neutrophils, ${ }^{17}$ it can be hypothesized that ADAM15 could facilitate the transepithelial migration of neutrophils through a mechanism similar to that operating across the endothelial barrier in the absence of VE-cadherin.

In conclusion, this study delineates the involvement of ADAM15 and its binding partners in the cellular crosstalks in the setting of active inflammation, and in the balance between epithelial regeneration and differentiation during inflammatory bowel disease.

\section{Acknowledgements}

This work was in part supported by two grants 'Projet Hospitalier Régional de Recherche Clinique' BRD05/10/C and BRD04/6/P. Dr Merlin is supported by a grant from National Institutes of Health DK061941-02.

\section{References}

1 Weskamps G, Blobel CP. A family of cellular proteins related to snake venom disintegrins. Proc Natl Acad Sci USA 1994;91:2748-2751.

2 Wolsberg TG, Straight PD, Gerena RL, et al. ADAM, a widely distributed and developmentally regulated gene family encoding membrane proteins with a disintegrin and metalloprotease domain. Dev Biol 1995;169:378-383.

3 Black RA, White JM. ADAMs: focus on the protease domain. Curr Opin Cell Biol 1998;10:654-659.

4 Gaultier A, Cousin H, Darribere T, et al. ADAM13 disintegrin and cystein-rich domains bind to the second heparin-binding domain of fibronectin. J Biol Chem 2002;277:23336-23337.

5 Poghosian Z, Robbins SM, Houslay MD, et al. Phosphorylation-dependent interactions between ADAM15 cytoplasmic domain and src family protein tyrosine kinase. J Biol Chem 2001;277:4999-5007.

6 Howard L, Nelson KK, Maciewicz RA, et al. Interaction of the metalloprotease disintegrins MDC9 and MDC15 with two SH3 domain-containing proteins endophilin 1 and SH3PX1. J Biol Chem 1999;348:21-29.

7 Bohm BB, Aigner T, Gerhsitz A, et al. Up-regulation of MDC15 (metargidin) messenger RNA in human osteoarthritic cartilage. Arthritis Rheum 1999;42:19461950.

8 Bohm BB, Aigner T, Roy B, et al. Homeostatic effects of the metalloproteinase disintegrin ADAM15 in degenerative cartilage remodeling. Arthritis Rheum 2005;52: 1100-1109.

9 Herren B, Raines EW, Ross R. Expression of a disintegrin-like protein in cultured human vascular cells and in vivo. FASEB J 1997;11:173-180.

10 Martin J, Eynstone LV, Davies M, et al. The role of ADAM15 in glomerular mesangial cell migration. J Biol Chem 2002;277:33683-33689.

11 Kratzschman J, Lum L, Blobel CP. Metargidin, a membrane anchored metalloprotese-disintegrin protein with a RGD integrin-binding sequence. J Biol Chem 1996;271:4593-4596.

12 Nath D, Slocombe PM, Stephens PE, et al. Interaction of metargidin (ADAM15) with $\alpha \mathrm{v} \beta 3$ and $\alpha 5 \beta 1$ integrins on different hematopoietic cells. J Cell Sci 1999; 112:579-587.

13 Eto K, Puzon-McLaughlin W, Sheppard D, et al. RGDindependent binding of integrin $\alpha 9 \beta 1$ to the ADAM12 and 15 disintegrin domains mediates cell-cell interactions. J Biol Chem 2000;275:34922-34930.

14 Ham C, Levkau B, Raines EW, et al. ADAM15 is an adherens junction molecule whose surface expression can be driven by VE-cadherin. Exp Cell Res 2002; 279:239-247.

15 Fenoglio-Preiser CM, Noffsinger A, Stemmermann GN, et al. Gastrintestinal pathology. An Atlas and Text, 2nd edn. Lippincott-Williams \& Wilkins: Philadelphia, PA, 1999.

16 Podolsky DK. Inflammatory bowel disease. N Engl J Med 2002;347:417-429.

17 Kucharzik T, Walsh SV, Chen J, et al. Neutrophil transmigration in inflammatory bowel disease is associated with differential expression of epithelial intercellular junction proteins. Am J Pathol 2001;159: 2001-2009.

18 Schmitz H, Barmeyer C, Fromm M, et al. Altered tight junction structure cones to the impaired epithelial barrier function in ulcerative colitis. Gastroenterology 1999;116:301-309.

19 ANAES. Recommandations pour la cryoconservation de cellules et tissus tumoraux dans le but de réaliser des analyses moléculaires. Ann Pathol 2001;21:184-201.

20 Mason DY, Sammons RE. The labeled antigen method of immunenzymatic staining. J Histochem Cytochem 1979;27:832-840.

21 Graham RC, Karnovsky MJ. The early stages of absorption of horseradish peroxidase in the proximal tubules of mouse kidney: ultrastructural cytochemistry by a new technique. J Histochem Cytochem 1966;14:291-302.

22 Blanchot-Jossic F, Jarry A, Masson D, et al. Upregulated expression of ADAM17 in human colon carcinoma: co-expression with EGFR in neoplastic and endothelial cells. J Pathol 2005;206:156-163.

23 Lum L, Reid MS, Blobel CP. Intracellular maturation of the mouse metalloprotease disintegrin MDC15. J Biol Chem 1998;273:26236-26247.

24 Charrier L, Yan Y, Driss A, et al. ADAM-15 inhibits wound healing in human intestinal epithelial cell monolayers. Am J Physiol Gastroenterol Liver 2005; 288:346-353.

25 Powell DW, Mifflin RC, Valentich JD, et al. Myofibroblasts. I. Paracrine cells important in health and disease. Am J Physiol 1999;277C:1-9. 
26 Kaye GI, Lane N, Pascal RR. The pericryptal fibroblast sheath: replication, migration, and cytodifferentiation of a mesenchymal cell system in adult tissue. II. Fine structural aspects of normal rabbit and human colon. Gastroenterology 1968;54:852-865.

27 Kaye GI, Pascal RR, Lane N. The pericryptal fibroblast sheath: replication, migration, and cytodifferentiation of a mesenchymal cell system in adult tissue. III. Replication and differentiation in human hyperplastic and adenomatous polyps. Gastroenterology 1971;60: 515-536.

28 Podolsky DK. Mucosal immunity and inflammation. V. Innate mechanisms of mucosal defense and repair: the best offense is a good defense. Am J Physiol 1999; 277:6495-6497.

29 Brittan M, Hunt T, Jeffrey $\mathrm{R}$, et al. Bone marrow derivation of pericryptal myofibroblats in the mouse and human small intestine and colon. Gut 2002;50: $752-757$.
30 Herren B, Garton KJ, Coats S, et al. ADAM15 overexpression in NIH3T3 cells enhances cell-cell interactions. Exp Cell Res 2001;271:153-160.

31 Gresham HD, Goodwin JL, Allen PM, et al. A novel member of the integrin receptor family mediates ArgGly-Asp-stimulated neutrophil phagocytosis. J Cell Biol 1989;108:1935-1943.

32 Muller WA, Weigl S. Monocyte-selective transendothelial migration: dissection of the binding and transmigration phases by an in vitro assay. J Exp Med 1992; 176:819-828.

33 Allport JR, Muller WA, Luscinskas FW. Monocytes induce reversible focal changes in vascular endothelial cadherin complex during transendothelial migration under flow. J Cell Biol 2000;148:203-216.

34 Nash S, Stafford J, Madara JL. Effects of polymorphonuclear leukocyte transmigration on the barrier function of cultured intestinal epithelial monolayers. J Clin Invest 1987;80:1104-1113. 